\title{
Determination of Drugs and Metabolites in Raw Wastewater Using Liquid Chromatography-Mass Spectrometry
}

\section{Klein DM ${ }^{1 *}$, Pandey $\mathbf{G}^{2}$, Blackwell $\mathbf{B}^{1}$ and Sperry $\mathrm{K}^{2}$}

${ }^{1}$ Department of Environmental Toxicology, Institute of Environmental and Human Health, Texas Tech University, USA

${ }^{2}$ Forensics Institute, Texas Tech University, USA

\begin{abstract}
A previous study in Lubbock, TX detected the cocaine metabolite benzoylecgonine in wastewater entering the municipal wastewater treatment plant. This work was conducted using chemical derivatization followed by gas chromatography-mass spectrometry (GC-MS) determination. This is a report using liquid chromatography-mass spectrometry (LC-MS) for determination of drug metabolites isolated from wastewater in Lubbock, TX by solid phase extraction (SPE). Using the LC-MS method, chemical derivatization is eliminated and multiple analytes can be determined in a single run. The results obtained showed that several compounds from different drug classes were detected. Compounds observed included cocaine, heroin, tetrahydrocannabinol, amphetamine, and benzodiazepine derivatives.
\end{abstract}

Keywords: Sewage epidemiology; Liquid chromatography-mass spectrometry; Drugs; Pharmaceuticals; Metabolites; Wastewater.

\section{Introduction}

The abuse of drugs, both legal and illegal, is a worldwide problem [1]. Several different approaches to characterizing drug use have been proposed. These include population surveys, checks and updates of criminal records, and consumer interviews. One method that has been reported to help track drugs and their metabolic products is the analysis of wastewater [2].

Zuccato et al. has termed this approach 'sewage epidemiology.' Using this method, it is possible to gain information about the range of substances that might be a problem in a community. It is also possible to develop some temporal information about when drugs are taken by sampling wastewater at particular intervals. Sewage epidemiology has been used in several countries [3-5].

A previous study was undertaken to examine the sewage epidemiology approach in Lubbock, TX [6]. The cocaine metabolite, benzoylecgonine was determined by gas chromatography - mass spectrometry (GC-MS). However, in this study only a single compound was monitored because it had to be isolated from the wastewater, purified, and chemically derivatized in order to be amenable to GCMS determination. Thus, GC-MS limits the number and type of compounds that can be included in a study.

The purpose of this study was to demonstrate the use of an ion trap based liquid chromatography-mass spectrometer (LC-MS) for the determination of drugs and drug metabolites in wastewater from Lubbock (TX). Unlike GC-MS, LC-MS does not require chemical derivatization of drug compounds. The use of LC-MS for the determination of pharmaceuticals products in wastewater has been previously reported [7]. In that case, several different classes of compound were determined including cocaine, opiates, cannabinoids and amphetamine compounds. Another advantage of LC-MS is that both parent compounds and metabolites can be determined in the wastewater samples.

This study employed an ion trap LC-MS. This is one of the least expensive configurations to use for this investigation. While other types of mass spectrometer may have greater sensitivity, the cost of these systems is prohibitive for most law enforcement groups. Further, the information derived from this study was used in forensic study, not the treatment and removal of the compounds where water may be further purified for reuse as drinking water. This allowed the work to be done on the least expensive platform and still have adequate sensitivity to detect compounds of interest.

In Lubbock (TX) the LC-MS was method was used to determine several classes of drugs and metabolites found in wastewater with a single isolation and purification scheme that does not require chemical derivatization [8]. By working with the local forensic science community, it was possible to select several different classes of compounds that were of interest [9]. This study is an investigation of representative compounds to determine which if any were present in the Lubbock (TX) wastewater. From this work we are expanding the concept to use in other law enforcement situations such as prisons where monitoring of wastewater would provide rapid information about the use of illegal drugs in this situation.

The use of LC-MS to monitor drugs of abuse in wastewater provides a clear way to have forensic evidence obtained. The method requires only minimal sample clean-up and rapid determination of compounds of interest.

\section{Materials and Methods}

All drug standards and reference compounds (Table 1) were purchased from Cerilliant (Austin, TX). Standards were stored at $-20^{\circ} \mathrm{C}$. Methanol (LC-MS grade) was purchased from Honeywell Burdick \& Jackson (Morristown, NJ) and acetic acid was purchased from Fisher Scientific (Fair Lawn, NJ). $18 \mathrm{M} \Omega$ water was purified using a Barnstead system. A Kinetex $5 \mu \mathrm{C}-18100 \AA$ column $(150 \mathrm{~mm} \times$ $4.6 \mathrm{~mm}$ ) was used for separation of analytes. Strata $\mathrm{X}^{\bullet} 33 \mu$ polymeric

*Corresponding author: David Klein, Ph.D., HCLD, Associate Professor Department of Environmental Toxicology, Institute of Environmental and Human Health, Texas Tech University, 1207 South Gilbert Drive, USA, Tel: 806-834-6044; E-mail: david.klein@ttu.edu

Received December 27, 2014; Accepted January 20, 2015; Published January 25,2015

Citation: Klein DM, Pandey G, Blackwell B, Sperry K (2015) Determination of Drugs and Metabolites in Raw Wastewater Using Liquid Chromatography-Mass Spectrometry. J Forensic Res 6: 268. doi:10.4172/2157-7145.1000268

Copyright: $\odot 2015$ Klein DM, et al. This is an open-access article distributed unde the terms of the Creative Commons Attribution License, which permits unrestricted use, distribution, and reproduction in any medium, provided the original author and source are credited. 
Page 2 of 5

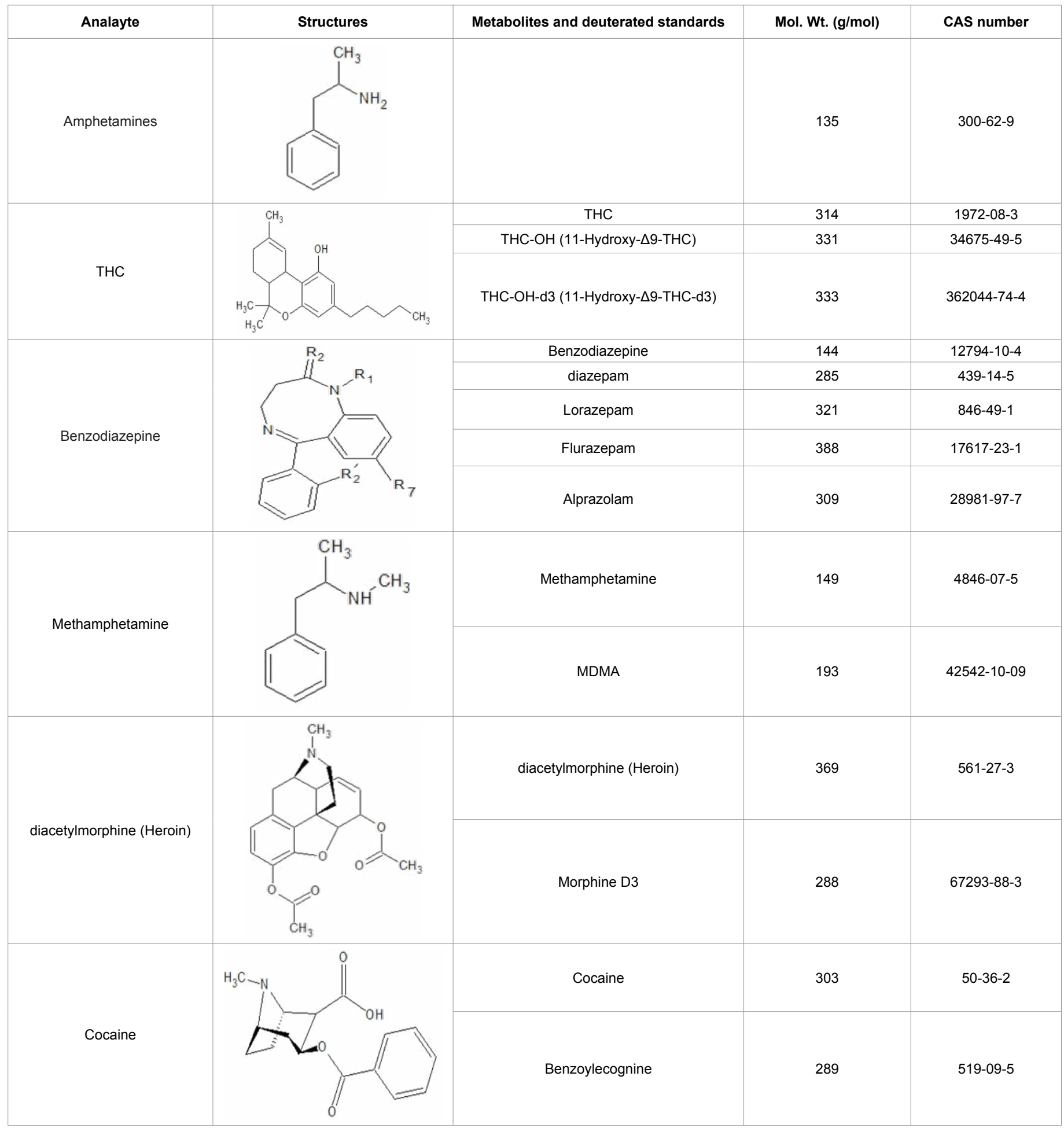

Table 1: Drug standards and reference compounds, Note. Mol. Wt.=Molecular weight; CAS number=Chemical Abstracts Service registry number.

reversed phase $(200 \mathrm{mg} / 6 \mathrm{~mL})$ solid phase extraction (SPE) cartridges were used to separate the analytes from the sample matrix. The column and SPE cartridges were obtained from Phenomenex (Torrance, CA).

Samples were obtained from the Lubbock Wastewater Treatment Plant (LWWTP). This is the only site in the city of Lubbock for wastewater treatment. Both weekday and weekend samples were collected and all samples were obtained in the morning using amber glass collection bottles. The collected samples were adjusted to $\mathrm{pH} 2$ using $37 \%$ Hydrochloric acid, and were stored in dark at $4^{\circ} \mathrm{C}$ until they were processed. It was found in a previous study that $\mathrm{pH}$ adjustment was necessary to prevent degradation of the analytes during storage; $\mathrm{pH}$ adjusted samples were found to be stable for one month [6].

The wastewater samples were held stable in order to allow particulate matter to settle to the bottom of the container. The water 
was then decanted and $500 \mathrm{~mL}$ samples were filtered using $1.2 \mu$ glass filters (Whatman). SPE was conducted using cartridges conditioned with $6 \mathrm{~mL}$ methanol followed by $6 \mathrm{~mL}$ water. The filtered samples were applied to the cartridges and the cartridges were washed with ultrapure water and subsequently dried with $\mathrm{N}_{2}$ for $30 \mathrm{~min}$. Elution of the compounds was performed with $6 \mathrm{~mL}$ of methanol. The eluate was evaporated to near dryness under a gentle stream of $\mathrm{N}_{2}$. The residue was re-dissolved in $1 \mathrm{~mL}$ of $\mathrm{H}_{2} \mathrm{O} /$ Methanol $(9 / 1 ; \mathrm{v} / \mathrm{v})$ containing $0.1 \%$ acetic acid. $100 \mathrm{ng}$ of deuterated standard (hydroxy THC-d3) was added to each sample as a surrogate.

LC-MS was conducted using a Surveyor LC, Thermoelectron, USA) interfaced to an ion trap mass spectrometer (Thermo Finnigan, LCQ Advantage, USA) equipped with an electrospray ionization source. The chromatographic conditions are shown in Table 2. Tandem mass spectrometry (MS/MS) was used to identify the compounds in this study. A full scan of the compounds of interest was run to determine the retention times of all the compounds shown in Table 3. This table provides information about the compounds selected for this study. It should be noted that this is just a representative group of compounds on interest. The number of compounds could be expanded greatly for application to other areas or concerns.

The selected reaction monitoring (SRM) MS/MS used the positive ionization mode. The capillary temperature was maintained at $300^{\circ} \mathrm{C}$. Auxiliary gas was $\mathrm{N}_{2}$ and Ar was applied as collision gas. The intensity of these gases and the collision energy varied based on an optimized tuning conducted prior to analysis for each compound. The run was divided into segments and the compounds each had individual scan events. The SRM was setup with the 1st segment to identify amphetamine, methamphetamine, and 3,4-methylenedioxymethamphetamine (MDMA), the 2nd segment had heroin, the 3rd segment had flurazepam, alprazolam, lorazepam, and diazepam with the last segment containing THC-OH (11-Hydroxy- $\Delta$ 9-THC) and THC-OH-d3 (internal standard surrogate compound). Cocaine and benzoylecgonine (BE) were run in a separate scan which had only 1 segment and 2 scan events for these two compounds.

It was not possible to obtain stable isotope-labeled standards for all of the compounds of interest to provide specific quantification for each component. The stable isotope-labeled hydroxy-THC-d 3 was used as a surrogate to measure ionization effects in the electro-spray LC-MS analysis.

\section{Results and Discussion}

Other researchers have reviewed the use of LC-MS for application in sewage epidemiology $[10,11]$. The current study was a survey of compounds of local interest to determine if ion trap LC-MS could detect the compounds of interest in Lubbock, Texas wastewater. Detections were observed from each of the classes of compounds of interest as seen in Figure 1. These results demonstrate measureable quantities of both drugs and metabolites in the wastewater inflow.

In this study, several compounds were detected in the samples by LC-MS (Table 4). While metabolic compounds were found in higher levels than the parent compounds, it was observed that parent drug of abuse compounds were present as well. This was a limited study and with specific interest and greater funding, more standards could be obtained to expand the number of compounds identified in the wastewater.

The results indicate that further investigation into sewage epidemiology is warranted in Lubbock, TX wastewater. One application of this method has been proposed using the method of sewage epidemiology to indicate if a prison has significant problems with drug use at their facility [12]. Texas has a large prison system and sewage epidemiology would be a very cost effective way for the state of Texas to monitor substance abuse in the prison system [13].

In order to more fully utilize these data, future work will include obtaining more metabolic compound standards and stable isotope internal standards. A review of the surrogate deuterium labeled hydroxyl-THC shows a considerable range of responses. In the laboratory, this work was repeated several times but the amount of material dissolved in the raw wastewater varies widely. Having stable isotopic internal standards for all compounds would be required for specific quantitation of each analyte.

Finally, the parent compounds are excreted in some cases. However, clearly benzoylecgonine occurs at some of the highest concentrations. Future studies will focus on metabolic break-down products which should be present at much higher concentrations than the parent compounds of interest.

\section{Conclusions}

The determination of several drugs of abuse, pharmaceuticals and associated metabolites was achieved with a simple extraction and sample clean up. Ion trap mass spectrometry was used successfully to determine these compounds in wastewater samples. The number of compounds of interest could readily be increased and determined if there is a need for this in other applications. The LC-MS has proven superior to GC-

\begin{tabular}{|c|c|c|}
\hline Column: & Phenomenex Kintex C-18 & \\
\hline Dimensions: & $5 \mu, 100 \mathrm{~A}, 150 \mathrm{~mm} \times 4.6 \mathrm{~mm}$ & \\
\hline Mobile Phase: & $0.1 \%$ acetic acid $(\mathrm{v} / \mathrm{v}) \mathrm{in} \mathrm{H}_{2} \mathrm{O}$ (Eluent A) & \\
\hline & $0.1 \%$ acetic acit $(\mathrm{v} / \mathrm{v})$ in $\mathrm{MeOH}$ (Eluent B) & \\
\hline Flow: & $1 \mathrm{~mL} / \mathrm{min}$ & \\
\hline Gradient: & $0 \% \mathrm{~B}$ to $10 \% \mathrm{~B}$ & $0-8 \mathrm{~min}$ \\
\hline & $10 \% \mathrm{~B}$ to $60 \% \mathrm{~B}$ & $8-15 \mathrm{~min}$ \\
\hline & $60 \% \mathrm{~B}$ to $70 \% \mathrm{~B}$ & $15-20 \mathrm{~min}$ \\
\hline & $70 \% \mathrm{~B}$ to $80 \% \mathrm{~B}$ & $20-25 \mathrm{~min}$ \\
\hline & $80 \% \mathrm{~B}$ & $25-30 \mathrm{~min}$ \\
\hline Injection Vol. & $80 \% \mathrm{~B}$ to $5 \% \mathrm{~B}$ & $30-35 \mathrm{~min}$ \\
\hline Column Temp: & $20 \mu \mathrm{L}$ & \\
\hline Sample Tray: & $35^{\circ} \mathrm{C}$ \\
\hline
\end{tabular}

Table 2: Chromatographic conditions.

\begin{tabular}{|c|c|c|c|c|}
\hline \multirow{2}{*}{ Sample (Reference) } & \multirow{2}{*}{ Retention Time } & \multirow{2}{*}{ Precursor } & \multicolumn{2}{|c|}{ Reaction lons } \\
\cline { 4 - 5 } & & & lon 1 & lon 2 \\
\hline Amphetamine & 8.75 & 136.1 & 119 & NA \\
\hline Methamphetamine & 10.07 & 150.2 & 119 & 91.2 \\
\hline MDMA & 11.39 & 194.1 & 163 & NA \\
\hline Heroin & 13.73 & 370.4 & 328.2 & 268.3 \\
\hline Cocaine & 13.74 & 304.1 & 182.1 & 150.3 \\
\hline Benzoylecognine & 14.3 & 290.2 & 272.1 & 168.1 \\
\hline Flurazepam & 15.75 & 388.1 & 315.3 & 288.3 \\
\hline Alprazolam & 19.08 & 309.3 & 281.2 & 274.3 \\
\hline Lorezepam & 19.09 & 321.2 & 275.5 & 302.9 \\
\hline Diazepam & 20.85 & 285.3 & 257.2 & 228.2 \\
\hline THC-OH & 27.51 & 331.2 & 313.1 & NA \\
\hline THC-OH-d3 & 27.45 & 334.2 & 316.2 & NA \\
\hline
\end{tabular}

Table 3: The retention times of all the compounds, Note: The information was obtained by running the standard mix of the compounds and tuning the compounds in the LC-MS instrument. NA represents that there was only 1 reaction ion for the compound. 


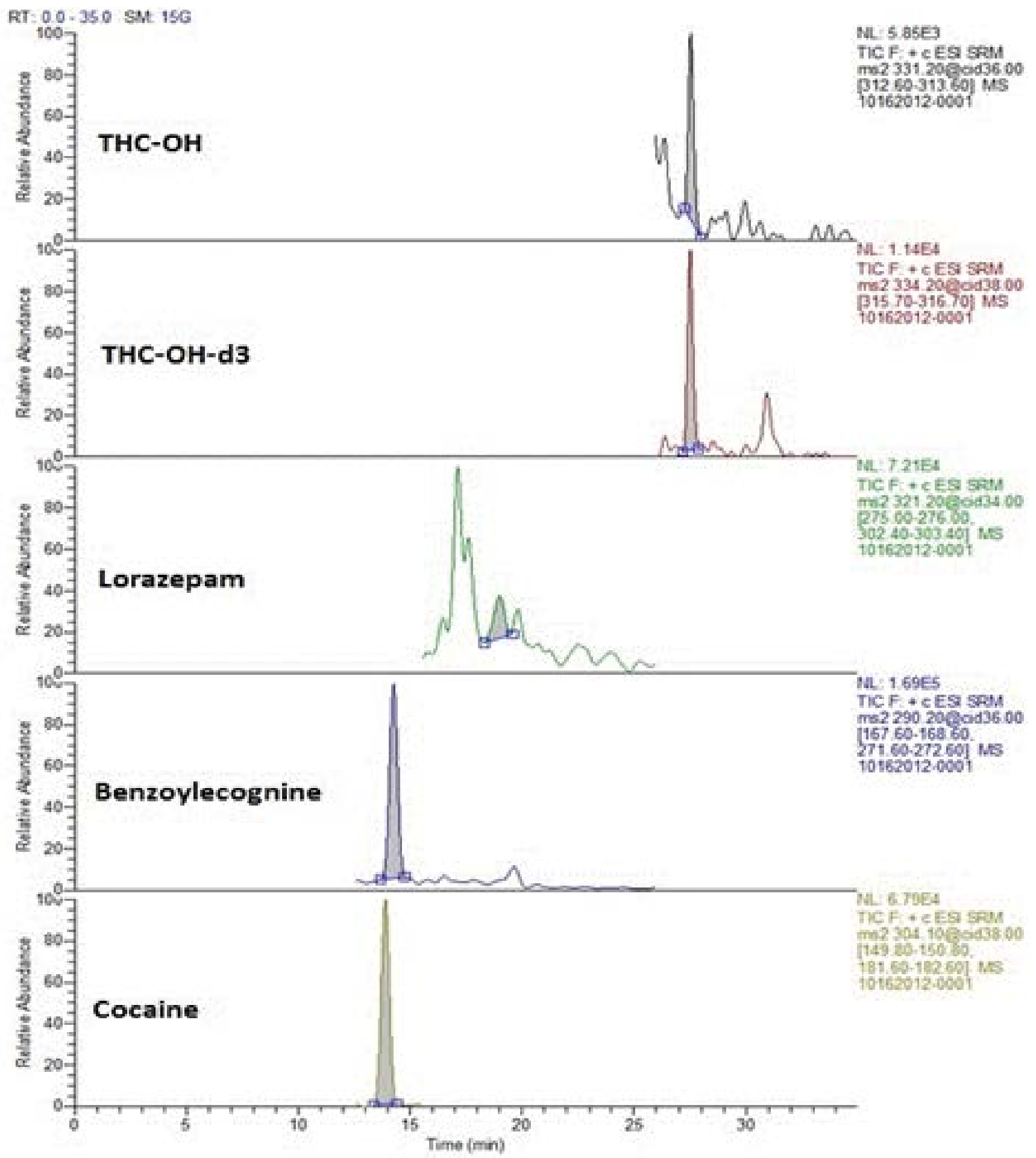

Figure 1: Drugs and metabolites identified in raw wastewater using liquid chromatography-mass spectrometry.

MS in ease of sample preparation and the number of compounds that can be determined simultaneously. Overall, this method is appropriate for use in forensic applications without requiring the more expensive instrumentation that is employed for residual analysis in water that is going to be routed for reuse and drinking water.

\section{Acknowledgement}

The authors would like to thank the Lubbock Water Reclamation Plant for access and assistance in sample collection as well as Prof. Todd Anderson who reviewed an earlier version of this manuscript. 
Citation: Klein DM, Pandey G, Blackwell B, Sperry K (2015) Determination of Drugs and Metabolites in Raw Wastewater Using Liquid ChromatographyMass Spectrometry. J Forensic Res 6: 268. doi:10.4172/2157-7145.1000268

Page 5 of 5

\begin{tabular}{|c|c|c|c|c|c|c|}
\hline Drug Compound & & Weekday Sample & & Weekend Sample & & \\
\hline & Collection & Presence & Response & Collection & Presence & Response \\
\hline \multirow[t]{2}{*}{ Amphetamine } & 1st & - & NA & 1st & - & NA \\
\hline & 2nd & + & 816 & 2nd & - & NA \\
\hline \multirow[t]{2}{*}{ Methamphetamine } & $1 \mathrm{st}$ & - & NA & $1 \mathrm{st}$ & + & 4770 \\
\hline & 2nd & - & NA & 2nd & + & 4720 \\
\hline \multirow[t]{2}{*}{ MDMA } & 1st & + & 370 & 1st & - & NA \\
\hline & 2nd & + & 673 & 2nd & - & NA \\
\hline \multirow[t]{2}{*}{ Heroin } & 1st & + & 1380 & 1st & - & NA \\
\hline & 2nd & - & NA & 2nd & - & NA \\
\hline \multirow[t]{2}{*}{ Cocaine } & $1 \mathrm{st}$ & - & NA & 1st & + & 331000 \\
\hline & 2nd & + & 187000 & 2nd & + & 411000 \\
\hline \multirow[t]{2}{*}{ Benzoylecognine } & $1 \mathrm{st}$ & + & 389000 & $1 \mathrm{st}$ & + & 834000 \\
\hline & 2nd & + & 421000 & 2nd & + & 1000000 \\
\hline \multirow[t]{2}{*}{ Flurazepam } & 1st & - & NA & $1 \mathrm{st}$ & - & NA \\
\hline & 2nd & - & NA & 2nd & - & NA \\
\hline \multirow[t]{2}{*}{ Alprazolam } & 1st & + & 2550 & $1 \mathrm{st}$ & - & NA \\
\hline & 2nd & + & 2650 & 2nd & + & 7900 \\
\hline \multirow[t]{2}{*}{ Lorezepam } & 1st & + & 44800 & 1st & + & 44600 \\
\hline & 2nd & + & 30500 & 2nd & + & 30800 \\
\hline \multirow[t]{2}{*}{ Diazepam } & $1 \mathrm{st}$ & - & NA & $1 \mathrm{st}$ & - & NA \\
\hline & 2nd & - & NA & 2nd & - & NA \\
\hline \multirow[t]{2}{*}{$\mathrm{THC}-\mathrm{COOH}$} & $1 \mathrm{st}$ & + & 2630 & 1st & + & 1910 \\
\hline & $2 n d$ & + & 17400 & $2 n d$ & + & 14000 \\
\hline \multirow[t]{2}{*}{ THC-OH d3 } & $1 \mathrm{st}$ & + & 16300 & 1st & + & 32900 \\
\hline & 2nd & + & 35500 & $2 n d$ & + & 64500 \\
\hline
\end{tabular}

Table 4: Compounds detected in the samples by LC-MS, Note: '+'=Present; '-'=Absent, Weekday sample 1st=Tuesday, 2nd=Thursday , Weekend samples are all Staken on Saturday.

\section{References}

1. David $H$ (2012) Perscription drug addiction: the treatment challenge. The Lancet 379: 17-18.

2. Zuccato E, Chiabrando C, Castiglioni S, Calamari D, Bagnati R, et al. (2005) Cocaine in surface waters: A new evidence-based tool to monitor community drug abuse. Environmental Health: A Global Access Science Source 4: 26-33.

3. Baker DR, Oscenaskova V, Kvicalova M, Kasprzyk-Hordern B (2012) Drugs of abuse in wastewater and suspended particulate matter - Further developments in sewage epidemiology. Environment International 48: 28-38.

4. Berset JD, Brennneisen R, Mathieu C (2010) Analysis of licit and illicit drugs in waste, surface and lake water samples using large volume direct injection high performance liquid chromatography - Electrospray tandem mass spectrometry (HPLC-MS/MS). Chemosphere 81: 859-866.

5. Khan U, Nicell JA (2011) Refined sewer epidemiology mass balances and their application to heroin, cocaine and ecstasy. Environmental International 37: 1236-1252.

6. Kinyua J, Anderson TA (2012) Temporal analysis of the cocaine metabolite benzoylecognine in wastewater to estimate community drug use. Journal of Forensic Science 57: 1349-1353.
7. Postigo C, Lopez de Alda MJ, Barcelo D (2008) Analysis of drugs of abuse and their human metabolites in water by LC-MS2: A non-intrusive tool for drug abuse estimation at the community level. Trends in Analytical Chemistry 27 1053-1069.

8. Pandey G (2012) Estimation of Illicit Drug Use by Analysis of Raw Wastewater Using LC-MS. Lubbock, TX: Texas Tech University.

9. Sperry Kathy (2012) Director, Forensic Science Institute, Texas Tech University.

10. Hayley E. Jonesa, Matthew Hickmana, Barbara Kasprzyk-Hordernb, Nicky J. Weltona, David R. Bakerc, et al. (2011) Illicit drug consumption estimations derived from wastewater analysis: A critical review. Science of the Total Environement 409: 3564-3577.

11. van Nuijs ALN, Castiglioni S, Tarcomnicu I, Postigo C, Lopez de Alda M, et al. (2008) Analysis of cocaine and its principal metabolites in waste and surface water using solid-phase extraction and liquid chromatography-ion trap tandem mass spectrometry. Analytical and Bioanalytical Chemistry 391: 1309-1319.

12. Postigo C, de Alda ML, Barceló D (2011) Evaluation of drugs of abuse use and trends in a prison through wastewater analysis. Environ Int 37: 49-55.

13. Window on State Government. 